December, 1995

UTAS-PHYS-96-03

hep-th/yymmdd

\title{
Chord diagrams and BPHZ subtractions
}

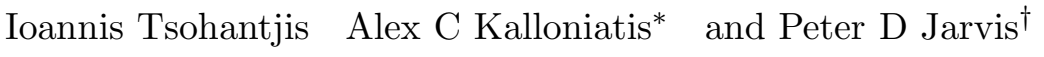 \\ Department of Physics, University of Tasmania \\ GPO Box 252C Hobart, Australia 7001
}

\begin{abstract}
The combinatorics of the BPHZ subtraction scheme for a class of ladder graphs for the three point vertex in $\phi^{3}$ theory is transcribed into certain connectivity relations for marked chord diagrams (knots with transversal intersections). The resolution of the singular crossings using the equivalence relations in these examples provides confirmation of a proposed fundamental relationship between knot theory and renormalization in perturbative quantum field theory.
\end{abstract}

\footnotetext{
* Institute for Theoretical Physics III, University of Erlangen-Nuremberg, D-91058 Erlangen, Germany

† Alexander von Humboldt Fellow email: Peter.Jarvis@phys.utas.edu.au
} 


\section{Introduction and Discussion}

Despite the predictive successes of quantum field theory established over the last half century since the formulation of the renormalization prescription, the mathematical well-foundedness of the procedure has been questioned since its very inception. As is well known, alternative approaches such as axiomatic, $S$-matrix and ultimately string theories have been spawned as a result. On the other hand, recent study of low-dimensional quantum field theory $(D=2$ or 3) has established deep connections with mathematics and geometry. In this note we consider whether such developments can be related to the traditional renormalization programme of perturbative quantum field theory $(p \mathrm{QFT})$ in arbitrary dimensions. Recently Kreimer [1, 2, 3] and Kreimer and Broadhurst 4 have proposed a relationship between Feynman graphs in $p \mathrm{QFT}$ and knot theory, developed in terms of skein relations on link diagrams associated with the Feynman graphs. Here we attempt to provide an alternative concrete formulation, as well as formal confirmation, of these ideas. Specifically, for a class of ladder-type vertex corrections in $\phi^{3}$ theory, we show how the graphs' contributions to the renormalization constant, including the correct counterterm subtractions, can be obtained by using equivalence relations on associated chord and singular knot diagrams.

The class of Feynman graphs considered here is that of vertex corrections in the ladder approximation, in which the rungs are simple or crossed propagators. Below, we firstly note $(\S 2)$ that such graphs have a direct interpretation in terms of marked chord diagrams (5, 6, (7). Each such diagram can be mapped to a knotted loop, including transversal intersection points[6, 9]. Then, motivated by the combinatorics of the BPHZ[8] subtraction scheme, and features of the types of divergent integrals which arise for the graphs [1, 2, 3], we formulate equivalence relations between such diagrams. The result of the iterative application of the rules is an expression for the value of the divergent part of each graph, plus its counterterm subtractions, which gives the graph's contribution to the renormalisation constant at the appropriate order. The procedure is illustrated with several examples $(\S 3)$. These clarify the suggestion [1, 2, 33, 迎 that certain types of Feynman graph 'topology', contributing to the renormalization constant with transcendental number coefficients, should be associated with knots. Finally, a more abstract, algebraic setting for the transcription of Feynman graphs into the language of knots is given $(\S 4)$. In our concluding remarks we suggest how the proposed formalism can be extended to arbitrary Feynman graphs and divergences, and speculate on the implications of the work for the significance of the renormalization procedure in $p \mathrm{QFT}$.

\section{Marked chord diagrams and counterterm subtractions}

In the BPHZ approach [8] to renormalization, the regularization of a given Feynman graph $\gamma$ is specified by of a sum of subtracted terms $\gamma \backslash \Gamma \cdot \prod_{\alpha \in \Gamma} \mathcal{T}(\alpha)$ generated by disjoint families $\Gamma=\left\{\alpha_{1}, \alpha_{2}, \ldots\right\}$ of divergent subgraphs. The explicit construction involves specific operations on the respective integrands (Taylor expansions with respect to external momenta); nevertheless the combinatorics should be seen in terms of operations on the graph $\gamma$ as a whole (indeed, the additional terms correspond to the counterterm subtractions in the usual formulation involving renormalization constants). It is at this level that the transcription into the language of manipulating singular knot diagrams should be seen.

We deal with $\phi^{3}$ theory, and for the present consider only corrections to the basic one loop vertex graph in the ladder approximation, where only the internal rungs (propagators) may be crossed, and no self-energy graphs, or dressings of internal propagators or vertices, are allowed. The formalism to be developed requires for its starting point that the Feynman graph 
be representable as a chord diagram [5, 6, 7]. For this class of ladder corrections the transcription is immediate: the original loop serves as the circle on which all vertices are located, and the internal propagators are viewed as chords (which may cross). In order to retain knowledge of the external vertices these are marked with ticks on the appropriate external arcs. Examples of Feynman graphs and their chord transcriptions, together with some graphs which are not admitted for present purposes, are given in Figure 1. Obviously, by restricting ourselves to this class of diagrams, we avoid issues of two-point and overlapping divergences. It should be emphasized that the discussion is intended simply to illustrate the working assumption that it is useful, at least for renormalization purposes, to associate Feynman graphs to knots we do not attempt a derivation of this association. However, for a more abstract setting for the formalism, and consideration of possible extensions to arbitrary Feynman graphs, see the concluding remarks below.

Having arrived at a chord characterisation $D_{\gamma}$ of the Feynman graph $\gamma$, we associate it [6] with an oriented singular (framed) knot $K_{\gamma}$, where the singularities correspond to transverse self-intersection points, represented as filled nodes. This is done by traversing the circle in a given sense (say, anti-clockwise), and associating with each chord a crossing point, so that the two ends of each chord are identified. Since these are the only allowed self-intersections, the necessity for these to be consistent will generally force a choice at several other places as to which arcs over or undercross, so that there is in principle a family of possibilities (marks are transferred to the appropriate arcs). Some examples are given in Figure 2, together with an extended notation for the nonstandard examples wherein marked crossings are introduced and represented by unfilled nodes.

The basic rules by which we propose the knot diagrams are to be manipulated are the connectivity relations $I, I I$, described in Figure 3. Here ' 1 ', '2' are understood to be parts which remain unchanged wherever they appear, whose connections with the remainder occur smoothly at the boundaries. The connectivity properties allow the singularities to be resolved in terms of conventional over- and under- crossings. The standard discussion[6] disallows isolated chords (so that the left-hand side of $I$ would vanish). Here however it is precisely the more involved relations $I, I I$ which generate the counterterm subtractions. Both ensure that two-line reducible knots can be simplified in terms of indecomposable parts (reflecting the subdivergence structure of the original graph). An additional essential notion is that of 'bracketing' $\langle\cdot\rangle$, which is allowed to any (finite) recursive depth (see below). Also, each of the disconnected parts ' 1 ', '2' inherits the other's additional markings, in order that it be regarded as a legitimate three-point contribution (the distinction between ' 1 ' and ' 2 ' is determined by which side carries the double marking (this may be distributed between different arcs or propagators as in the last examples of Figures 1, 2).

Each of $I, I I$ involves an unbracketed term on the right hand side, in which the transversal crossings are either dissolved by smoothing (as in $I$ ) or resolved by specifying over- and undercrossings $(I I)$. On the ' 2 ' term there appear additional twists on the closing loop, corresponding to an increase of framing number (in the blackboard framing) equal to the grading of ' 1 ' (the total number of nodes on the ' 1 ' side, including the explicitly drawn ones). Further, in the bracketed term of $I I$, ' 1 ' is closed not by an additional loop, but by a marked vertex (represented by an unfilled node) formed by fusing the original transversal crossings, with the elimination of two of the original six connecting lines to the remainder (in the same way, $I$ can be thought of as a special case of $I I$ in which the two crossings are fused, with the small connecting arcs cancelling each other out). Which lines survive appears to depend on the details of these connections, but can be established for particular examples (see $\S 3$ below). Finally, it will be apparent from these examples that the rules must be applied in a particular order to the diagrams' two-line reducible 
locations (working left to right as drawn in the figures), and further that the bracket on the right-hand side embraces not only the ' 1 ' part but also any additional disconnected terms which multiply the expression on the left-hand side. The necessity to refer back to the original BPHZ combinatorics for these points can be expected to be overcome in a more complete treatment (see concluding remarks below).

The result of the application of the rules to a given knot $K_{\gamma}$ is an expression involving products of bracketed and unbracketed framed knots. Following Kreimer [1, 2, 3, 4, the central claim is that the divergent contribution of the original Feynman diagram $\gamma$, including counterterm subtractions if any, has precisely this structure, where there is a systematic association between the component parts and certain basic divergent integrals. The (iterated) brackets signal taking divergent parts (see (3) below) with respect to the regularization parameter, and the projection on to the divergent piece of the overall result is the graph's contribution to the renormalization constant. (Strictly speaking, the renormalization point, and the precise dependence on momentum scale, should be spelled out. For the present sketch of the combinatorics we work with a 'minimal subtraction' type scheme[1], but the details are not required).

\section{$3 \quad$ Examples}

We illustrate the technique with some examples (see Figure 1 ) before giving a more abstract discussion. The first case in the figure shows the result for the case of simple ladder corrections to the vertex (corresponding to the first entry of Figure 1 and 2) at three loops; higher loops follow in the same way. Obviously only relation $I$ need be applied, and the result is a sum of terms involving bracketed and unbracketed products of simple unknotted loops with framing number increasing up to two. This accords precisely with the algebraic contribution of this graph to the renormalization constant when the subtractions are taken into account. The basic three loop graph is accompanied in the sum over disjoint families of divergent subgraphs $(\gamma \backslash \Gamma)$ by the one and two loop counterterms $\left(\prod_{\alpha \in \Gamma} \mathcal{T}(\alpha)\right)$ corresponding to the bracketed pieces in the figure. Only massless propagators contribute (infrared divergences being avoided in the presence of the subtractions), and as pointed out in [1, at least in dimensional regularization, the multiloop divergent integrals possess an iterative kernel structure whereby the key ingredient is

$$
{ }_{k} \Delta=\left(q^{2}\right)^{(k+1) \varepsilon} \int d^{d} p \frac{\left(p^{2}\right)^{-k \varepsilon}}{p^{4}(p+q)^{2}}
$$

which by Lorentz invariance is independent of the direction of $q$ (note that we are working in $\phi_{D=6}^{3}$ ). The result is a product of such terms with rising values of $k$, represented graphically by the increase of framing (or writhe number in the blackboard framing) in the figure. Finally, the contribution of the graph to the renormalization constant, in direct correspondence with the diagrammatical terms, is

$$
<Z>=\left\langle{ }_{0} \Delta{ }_{1} \Delta{ }_{2} \Delta-{ }_{0} \Delta<{ }_{0} \Delta{ }_{1} \Delta>-{ }_{0} \Delta{ }_{1} \Delta<{ }_{0} \Delta>+{ }_{0} \Delta<{ }_{0} \Delta<{ }_{0} \Delta>>\right\rangle,
$$

where now $<\cdot>$ signifies taking the divergent part (in minimal subtraction).

The second and third examples in Figure 4 correspond to the second and third entries in Figures 1 and 2. In each case there is only a single divergent subgraph, so only two terms on the right hand sides appear. Now, however, the connectivity relation II must be applied (as indicated by the dotted shadings on the left hand sides). The bracketed terms specify the correct counterterms, namely the divergent pieces corresponding to the primitive two loop Feynman graphs with two and three crossed propagators (compare the fourth and fifth entries 
in Figures 1 and 22). Concerning the point mentioned above about the connections of the marked (unfilled) node to the rest of the diagram in the bracketed ' 1 ' term of the equivalence relation $I I$, it can be seen here how the rule is to be interpreted. Consider the path starting with the upper node, continuing to the lower node via what would have been the route through '2' (but now smoothed), and returning to to the upper node within ' 1 '. From these cases it appears that this entire line segment is eliminated, with the connections to the rest of the diagram being taken up by the replacement marked (unfilled) node. The markings are here given so as to agree with the needed counterterms, but with the elimination of the said line segment, it is not apparent what the general rule for reassigning the second mark might be. As emphasized at the outset, the answers arrived at here arise by explicit reference back to the BPHZ algorithm for these cases: in the absence of a transcription of arbitrary Feynman graphs into chord diagrams, the rule cannot be given in complete generality (see concluding remarks below about extensions of the present approach).

Let us examine the unbracketed terms in these examples. In the first case, there are only two singular crossings to be resolved, and there emerges for the ' 1 ' term a standard knot, a marked version of the trefoil (multiplied by the simple unknotted loop with the correct framing number). In the second case, one singular crossing remains in the ' 1 ' term. Clearly the additional entanglements engendered by $I I$ prevent the further application of $I$ to this remaining node. In general, the only possibilities for further simplification using $I$ or $I I$ should be where there is genuine two-line reducibility arising from other parts of the original Feynman graph.

In the case of the simple ladder graphs, the diagrammatic expression after applying $I, I I$ was noted above to be in direct correspondence with the algebraic structure of the contributions to the renormalization constant, and a mapping could be established between framed unknots and divergent one loop integrals, in agreement with [1, 2, 3, 4. For more complicated 'topologies', such as the second and third examples in Figure 1 (via Figures 1, 2) which we are considering, divergent integrals of increasing complexity are obviously involved. The central claim of [1, 2 , 3, 4 is that there is in all cases a systematic association between the algebraic structure and the results of diagrammatic manipulations (developed there in terms of skein relations on link diagrams associated with the Feynman graphs). The ubiquitous appearance of for example $\zeta(3)$ in certain three loop integrals is thus to be interpreted as a signal of the repeated occurrence of a particular diagrammatic term, claimed in fact to be the trefoil, with higher transcendentals coming with other knots (the simple ladder graphs, associated with unknots, being free of transcendentals of this type 1, 2, 3, 歫).

The second and third examples in Figure 1 which we are considering provide confirmation of these claims, from the viewpoint of the present approach. In fact, as noted already, the second example yields directly the trefoil, in accord with the occurrence of $\zeta(3)$ in the leading divergence. In the third example, however, not all the singular knot crossings are resolved, and this will certainly be the case in general. At this stage it should be pointed out that we have until now made no use of an additional property which is usually applied [6], namely the Vassiliev type equivalence relation, whereby singularities are resolved in terms of a difference between standard over- and under- crossings (given as $V$ in Figure 5). In fact this relation is already implicit in the translation between chord and knot diagrams: provided that one is evaluating a singular knot with a Vassiliev invariant of type $n$, it is immaterial whether under- or over-crossings are used in the specification of the avoided crossings (because the difference is a knot with $n+1$ singularities, on which the Vassiliev invariant vanishes). From this, we would expect $V$ to be involved in the detailed interpretation of our formalism; again, it should be borne in mind that we are building only on the limited examples at hand. For the moment, we can make use of this idea in interpreting the third example, and note that one part of the unbracketed knot 
under $V$ is certainly a version of the trefoil (possibly in a different projection, and with different marking on the arcs from the previous case). Similarly, the bracketed part (corresponding to the primitive divergence, the fifth entry in Figures 1 and 2) also has the 'topology' of the trefoil (in fact the corresponding $p$ loop Feynman graph with one propagator crossing successive rungs

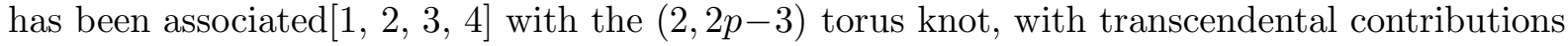
involving $\zeta(2 p-3))$.

\section{Abstract setting}

Up to now, we have presented a diagrammatic recipe in order to reproduce the combinatorics of the BPHZ subtraction scheme for a particular class of Feynman graphs. However, an algebraic setting (c.f. [6, 11, 12]) can be given which stands alone, and hopefully is general enough to encompass all graphs of the theory (see concluding remarks below). Firstly introduce the free module $\mathcal{D}$ of marked chord diagrams, given the structure of an abelian algebra by juxtaposition. The 'scalars' of this module comprise the ring $\Lambda$ of (one-sided) Laurent series in the regularization parameter (the cutoff momentum scale, or the $\varepsilon$ parameter in dimensional regularization with $d=D-2 \varepsilon)$. Inherent in this ring is the operation of the projection onto the divergent part: if $\lambda \in \Lambda$ we have

$$
\begin{aligned}
\lambda & =\frac{\lambda_{-N}}{\varepsilon^{N}}+\frac{\lambda_{-N+1}}{\varepsilon^{N-1}}+\cdots+\lambda_{0}+\lambda_{1} \varepsilon^{1}+\cdots, \\
<\lambda> & =\frac{\lambda_{-N}}{\varepsilon^{N}}+\frac{\lambda_{-N+1}}{\varepsilon^{N-1}}+\cdots+\lambda_{-1} .
\end{aligned}
$$

Correspondingly, the notion of bracketing is introduced as an abstract operation in $\mathcal{D}$ and in the associated algebra $\mathcal{A}$ of singular knots. The connectivity relations $I, I I$ generate an ideal $\mathcal{I}$ in $\mathcal{A}$. The implementation of the equivalence relations amounts to working in the factor algebra $\mathcal{P}=\mathcal{A} / \mathcal{I}$. $\mathcal{P}$ is generated as a (polynomial) algebra in the indecomposable parts and their bracketings, which consist of marked, framed knots $K_{i}$ (possibly including unresolved nodes; see examples and concluding remarks below). Thus, for the knot $K_{\gamma}$ associated with the chord diagram $D_{\gamma}$ for the Feynman graph $\gamma$, we have

$$
K_{\gamma}=\sum_{(i)(j)(k) \cdots} c_{(i)(j)(k) \ldots} K_{i_{1}} K_{i_{2}} \cdots<K_{j_{1}} K_{j_{2}} \cdots<K_{k_{1}} \cdots<\cdots>>\cdots>
$$

Finally, the renormalization constant contribution should be seen as the divergent piece of a homomorphism $Z$ from $\mathcal{P}$ to $\Lambda$ for which $Z\left(K<K^{\prime}>\right)=Z(K)<Z\left(K^{\prime}\right)>$ :

$$
\left.Z_{\gamma}=<Z\left(K_{\gamma}\right)>=\left\langle\sum_{(i)(j)(k)} c_{(i)(j)(k)} \Delta_{i_{1}} \Delta_{i_{2}} \cdots<\Delta_{j_{1}} \Delta_{j_{2}} \cdots<\Delta_{k_{1}} \cdots<\cdots>>\cdots\right\rangle\right\rangle
$$

where $Z\left(K_{i}\right)=\Delta_{i}$ are basic scalars (Laurent series) fixed once and for all in the theory (see the foregoing discussion in connection with the examples given above).

\section{Conclusions}

In this note we have exploited the possibility of interpreting a class of ladder diagrams in $\phi^{3}$ theory directly as chord diagrams, in order to present the BPHZ subtraction scheme in terms of equivalence relations on associated singular knot diagrams. The resulting structure is found to be 
present in the evaluation of the renormalization constant, if the various diagrammatic terms are taken to stand for algebraic contributions from basic divergent integrals. Moreover, the known association between certain diagram 'topologies' and transcendental number contributions to the renormalization constant (for example the occurrence of $\zeta(3)$ at three loops) is made more definite, in that the contributions from 'topologies' corresponding to indecomposable parts in the knot language, and as confirmed in the examples discussed, can in fact be associated with specific knots $(\zeta(3)$ with the trefoil, or $\zeta(2 p-3)$ with the $(2,2 p-3)$ torus knot [1, 2, 3, 俚).

Before speculating on the significance of the view of renormalization suggested in [1, 2, 2, 3, 4] and, supported and advocated more concretely by the present work, let us emphasize two major limitations of our approach. Firstly we have only been concerned with a simple class of ladder corrections to the three-point vertex, for which the chord transcription is immediate. In Figure 2 are given examples which do not comply - it is clear that in general, chords incorporating trivalent vertices are needed. All our conclusions are thus predicated on the assumption that moves can be introduced[6] whereby these more general cases can be reduced to the present framework. Secondly, we have of course ignored all considerations of two-point functions, and associated problems of overlapping divergences. In a sense, this second type of extension of the formalism poses less of a problem than the first, in that it can be envisaged how appropriate new equivalence relations $I I I, I V$ might be introduced to cover connectivity between two parts, each of which carries only one external marked line. Further issues, such as form factors (for example the distinction between mass and wave-function renormalizations in the two-point function), can be handled by a matrix formalism[1]. Work to handle these two major limitations, in order to give a complete picture of $\phi^{3}$ theory, is currently in progress.

Subject to these assumptions about the general validity of our work, the picture of $p \mathrm{QFT}$ afforded by our current approach is the following. Associated with each renormalizable theory are certain fundamental invariants $Z$ which, technically, belong to the algebraic dual of the algebra $\mathcal{P}$ of indecomposables introduced above (in the case of form factors they may be matrix valued over $\Lambda$ ). The contribution of each Feynman graph to the appropriate renormalization constant is then the infinite part of the evaluation of the corresponding $Z$ on the associated $K_{\gamma}$ as in (河) above, $Z_{\gamma}=\left\langle Z\left(K_{\gamma}\right)\right\rangle$ (or a component thereof in the case of form factors). Thus in order to evaluate $Z_{\gamma}$ it is in principle only a matter of expressing $K_{\gamma}$ in terms of equivalence classes in $\mathcal{P}$, and applying the homomorphism $Z$ assuming that its action is known on an algebraic basis for $\mathcal{P}$. Moreover, one may suggest further that the renormalization constant as a whole to all orders in $\hbar$, involving a summation over all Feynman graphs, has the significance of a true topological (manifold) invariant (although we do not speculate at this stage on the precise nature of such an invariant). This status would then accrue also to many of the standard functions of $p \mathrm{QFT}$ which depend on the renormalization process for their definition, such as the $\beta$ functions [10]. Of course, the characterization of the algebra $\mathcal{P}$ and its dual, and the construction of topological invariants therefrom may be a difficult mathematical problem (compare discussions of Conway algebras and skein quantization for arbitrary 3-manifolds [11, 12], the classification of Vassiliev invariants[6] or 3-manifold invariants [13]). Hopefully the present approach at least has the merit of pointing a way to a more fundamental view of the nature of renormalizable quantum field theories (in arbitrary dimensions), which may be explored in other contexts.

Note added in proof: It should be noted that, from the general $p$ loop result quoted, the interpretation of the third example in Figure 4 would be expected to involve the $(2,5)$ torus knot (associated with $\zeta(5)$ ). 


\section{Acknowledgements}

The authors would like to thank Dirk Kreimer for his insistence, during the course of his work in the theory group in Tasmania, that there should be a solid basis for his 'knottish' ideas, and for comments on a draft of this letter; and Hughan Ross for correspondence and alerting us to salient literature. We also thank Bob Delbourgo for constructive dialogue and healthy scepticism, other members of the group for their tolerance, and especially Neville Jones for computing support. Finally we acknowledge the Australian Research Council for a Small Grant project award which made possible the visits of ACK and GT.

\section{References}

[1] Dirk Kreimer, Knots and Divergences, Phys Lett B354 (1995) 117-124

[2] Dirk Kreimer, Renormalization and Knot Theory, preprint UTAS-PHYS-94-25 (hepth/9412045), to appear in J Knot Theory and its Ramifications

[3] Dirk Kreimer, Feynman Diagram Calculations - From finite integral representations to knotted infinities, in Proc IAHENP95 Conference (Pisa 1995), New Computing Techniques in Physics Research IV, Ed B Denby, D Perret-Gallix, (World Scientific)

[4] D J Broadhurst, D Kreimer, Knots and numbers in $\phi^{4}$ theory to 7 loops and beyond, Int J Mod Phys C6 (1995) 519

[5] M Alvarez and J M F Lambastida, Numerical knot invariants of finite type from ChernSimons perturbation theory, Nucl Phys B433 (1995) 555-596

[6] Dror Bar-Natan, On the Vassiliev Knot Invariants, Topology 34,2 (1995) 423-72

[7] Joan S Birman, New Points of View in Knot Theory, Bulletin (New Series) of the American Mathematical Society 28,2 (1993) 253-287

[8] See for example Claude Itzykson and Jean-Bernard Zuber, Quantum Field Theory, New York: McGraw-Hill (1980) Chap 8

[9] Louis H Kauffman, Knots and physics, Singapore: World Scientific (1991)

[10] D J Broadhurst, R Delbourgo, D Kreimer, Unknotting the polarized vacuum of quenched QED, preprint OUT-4102-60, UTAS-PHYS-95-40, MZ-TH/95-22 hep-ph/9509296)

[11] V G Turaev, Conway and Kauffman modules of a solid torus, Zap Nauch Sem LOMI 167 (1988) 79-89 (English translation: J Soviet Math)

[12] V G Turaev, Skein quantization of Poisson algebras of loops on surfaces, Ann scient Éc Norm Sup 24 (1991) 635-704

[13] R B Zhang and A L Carey, Quantum groups at odd roots of unity and topological invariants of 3-manifolds, preprint, Dept of Pure Mathenatics, University of Adelaide (1995) 

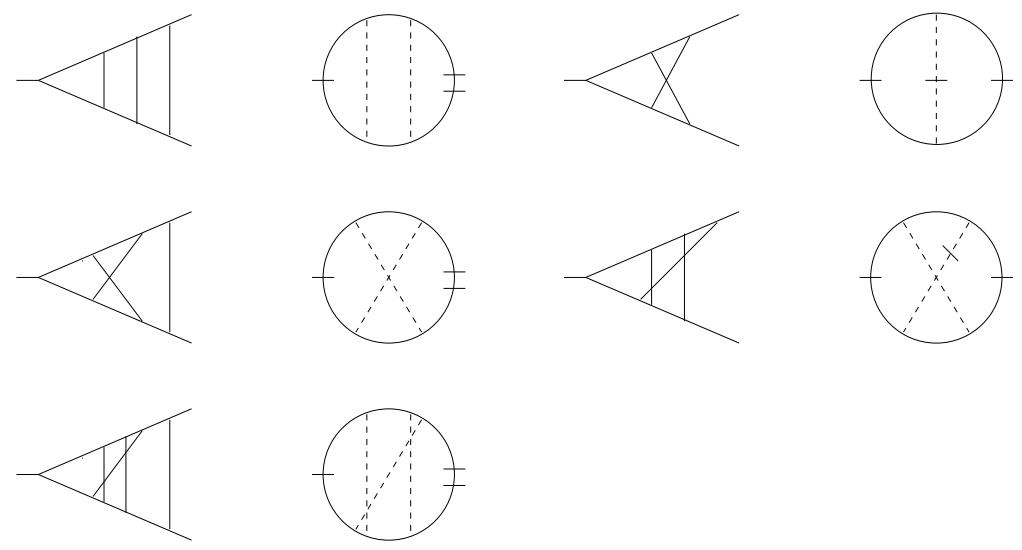

Figure 1: Examples of diagrams and their chord transcriptions. The last two are given by an extended notation in which a single internal propagator is marked. 

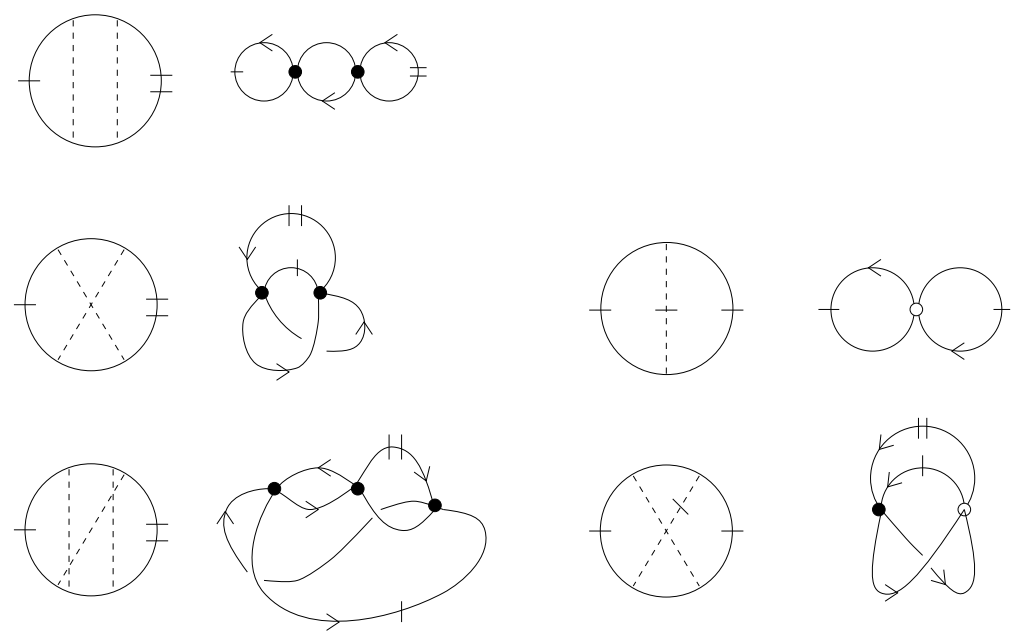

Figure 2: Examples of knots corresponding to the chords of Figure 1. The last two are handled by introducing unfilled nodes for the relevant knot crossings. 


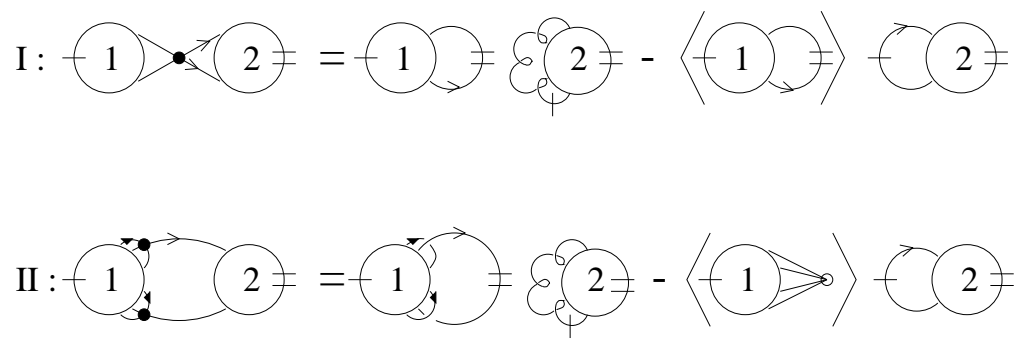

Figure 3: Rules $I, I I$ for manipulating singular knot diagrams derived from chords. The increase of framing number on the first ' 2 ' term on the right hand side is the number of nodes of the ' 1 ' part (including the explicitly drawn ones on the left hand side). For discussion of the unfilled nodes appearing in $I I$, see the text and the examples given in Figure 4 . 

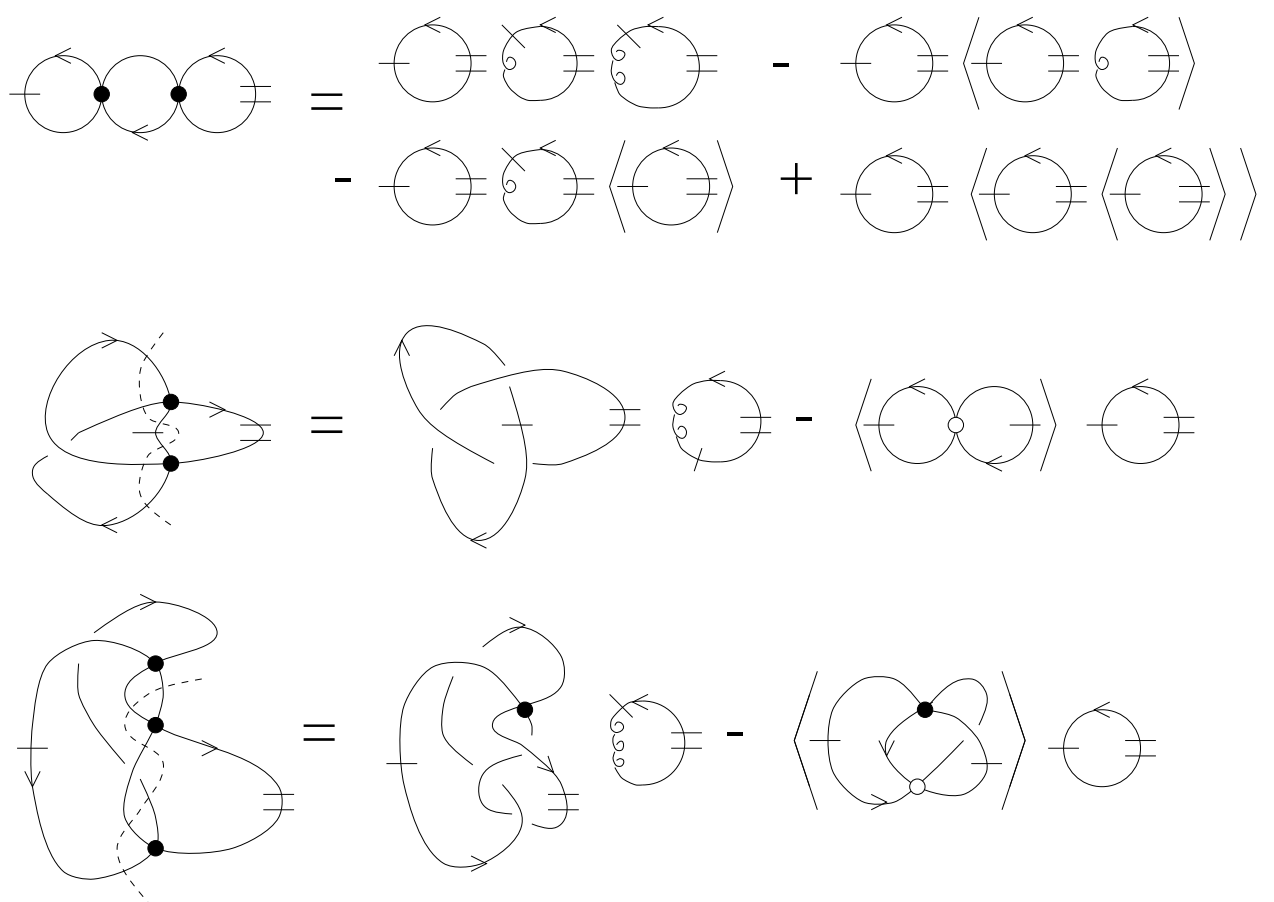

Figure 4: Examples of rules for manipulating knot diagrams derived from chords. In these cases the connections of the unfilled nodes are given explicitly (see Figure 3) 

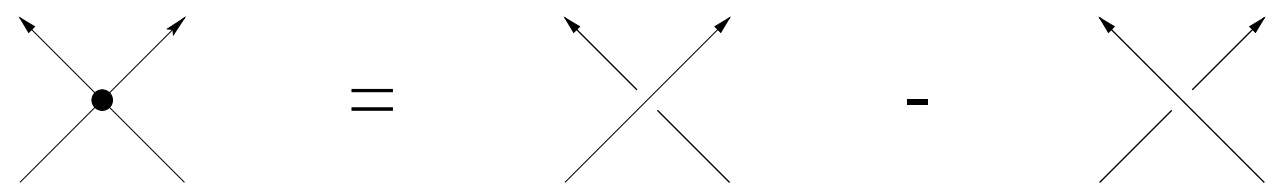

Figure 5: The Vassiliev property $V$ expressing a singular crossing as a difference between conventional over- and under- crossings. The complete set of equivalence relations would involve $I$, $I I$, together with new relations $I I I, I V$ covering two point divergences, as well as $V$. $V$ is used in the text in the interpretation of Figure 4 . 